\title{
Annotated checklist of Estonian bryophytes
}

\author{
Kai Vellak ${ }^{1,2}$, Nele Ingerpuu ${ }^{2}$, Mare Leis $^{3} \&$ Loore Ehrlich $^{4}$ \\ ${ }^{1}$ Natural History Museum, University of Tartu, 46 Vanemuise Street, Tartu 51014. E-mail: kai.vellak@ut.ee \\ ${ }^{2}$ Institute of Ecology and Earth Sciences, University of Tartu, 40 Lai Street, Tartu 51005. E-mail: nele.ingerpuu@ut.ee \\ ${ }^{3}$ Institute of Agricultural and Environmental Sciences, Estonian University of Life Sciences, 5 Fr. R. Kreutzwald Street, \\ 51014 Tartu. E-mail: mare.leis@emu.ee \\ ${ }^{4}$ Estonian Museum of Natural History, 26 Toompuiestee Street, 10148 Tallinn. E-mail: loore.ehrlich@loodusmuuseum.ee
}

\begin{abstract}
The updated list of Estonian bryophytes includes 594 species from all three phyla. Only one species is reported for Estonia according to the literature data, all others have voucher speciemens in herbaria, two of them outside of Estonia. Altogether 242 species are frequent in Estonia, 173 species are rare, and 161 are sporadically distributed. We do not have any recent data for 20 species, and their presence in Estonia is doubtful. In 2008 a new Estonian Red list was compiled and 369 bryophyte species were evaluated against IUCN criteria. Approximately one fifth of the Estonian bryoflora (129 species) is designated to the three threat categories.
\end{abstract}

Keywords: Bryoflora, frequencies of species, protected species, red list

\section{INTRODUCTION}

The diversity of taxa at the global or local scale depends greatly on the taxonomical research. The species number of bryophytes in the world varies between 15000 and 20000 (Shaw et al., 2011). The number of liverwort species, reported between 5500 and 9500 in recent 20 years, has been estimated to be 7500 by assessing rates of synonymy in recently revised taxa (Von Konrat et al., 2010). Regional species lists updated according to recent taxonomical conclusions are of help to understand the distribution of global diversity.

The first attempt to compile a list of bryophyte taxa occurring in Estonia dates back to the middle of the 19th century (Girgensohn, 1860). The first and most recent checklist that includes all groups of bryophytes was published in 1994 (Ingerpuu et al., 1994). Since then several species new for Estonia were recorded during inventories as well as herbarium material examinations (Kannukene et al., 1997; Leis $\&$ Kannukene, 2007; Vellak et al., 2001, 2006, $2009,2011,2013)$. The taxonomy of bryophytes has been changed during past 20 years and some species are separated into two or more species, some are united into one. Some subspecies have been raised to species level and vice versa. Taxonomical rearrangements have been made for higher levels as well. All this has led to the need to update the list of bryophytes registered in Estonia. The aim of this new checklist of Estonian bryophytes is to arrange the taxa according to recently accepted synonyms, supply every taxa with a voucher specimen, estimate the present frequency in Estonia and give proper names in Estonian for new taxa or new synonyms.

Nomenclature of species and lower taxa bases on the lists of European bryophytes (Hodgetts, 2015), for the higher ranks the Engler's system is used (Frey \& Stech, 2009). Some taxonomical supplements for hepatics are followed by Feldberg et al. (2010), Váňa et al. (2013), Shaw (2015) and Söderstöm et al. (2010, 2015), and for mosses Hedenäs \& Rosborg (2008), Spence (2005) and Werner et al. (2004).

\section{MATERIAL AND METHODS}

Four Estonian herbaria (TAA; TALL; TAM; TU), database eBiodiversity (http:/ / elurikkus.ut.ee/) and literature data were revised for updating the list. For every species, subspecies and variety a most typical, recent and abundant voucher specimen was chosen. The frequency of every species in Estonia was evaluated in three categories: 1) rare (r) species that have one to seven documented localities; 2) sporadically distributed (p) species that have 8 to 30 localities, and 3) frequent (fq) species that have 31 and more localities. Species occurrence were evaluated as doubtful (?) in Estonia if no records have been found since 1951. For the species that do not 
have any specimens in herbaria, the source reference is provided.

All new names in Estonian were discussed and confirmed by the Committee of Estonian Botanical Terminology.

\section{RESULTS AND DISCUSSION}

In 2015 the Estonian bryoflora includes 594 species. The list of species with their higher taxonomical ranks (genera, families, orders and classes), their common names and recent synonyms is available as an electronic supplement (http:/ /dx.doi.org/10.12697/fce.2015.52.14).

Previously three classes of the phylum Bryophyta were accepted, but according to the results of recent research it has been confirmed, that these classes are independent phyla (Vanderpoorten \& Goffinet, 2009). In Estonia all three phyla are presented: Anthocerotophyta includes two, Marchantiophyta 127, and Bryophyta 465 species. Numbers of orders, families and genera have increased, but numbers of subspecies and varieties have decreased (Table 1).

Compared with the previous list the Estonian bryoflora has increased by 84 species during last twenty years. From these 76 species are new findings for Estonia and sixteen species are results of new taxonomical combinations. Eight species are excluded from the previous list due to misidentifications or misinterpretation of synonymy.

Five species were excluded comparing with the previous list (Ingerpuu et al., 1994) due to misidentifications: specimens of Orthotrichum tenellum and Ditrichum heteromallum are now identified as $O$. pallens and D. flexicaule ac- cordingly (Kannukene et al. 1997), specimens of Dicranodontium denudatum and Dicranum muehlenbeckii as Ditrichum sp. and as Dicranum brevifolium (Vellak et al., 2009). Pterogonium gracile was first reported for Estonia in 1994 with two localities (Ingerpuu et al., 1994). Checking the specimens both were identified now as Pterigynandrum filiforme.

One species is eliminated from the list due to new taxonomical treatment. Despite good morphological characteristics, according to the molecular data Bryum neodamense has been found to be identical to Bryum pseudotriquetrum and they are treated as a single species (Holyoak \& Hedenäs, 2006). Pterogonium gracile and Bryum neodamense belong to the list of protected species of Estonia (Riigi Teataja, 2014a, b), but due to taxonomical re-arrangements they should be eliminated from that list.

Two further species (Pohlia campotrachela and Schistidium strictum) were eliminated from the list due to misinterpretation of synonymy. $P$. campotrachela was included in the previous list according to old records named as Bryum annotinum, collected by Girgensohn (Girgensohn, 1860), and as Pohlia grandiflora, reported by N. Malta (1930). These names are now accepted as Pohlia annotina. Unfortunately no herbarium specimens were found for Pohlia grandiflora, whereas Bryum annotinum from Girgensohns' collection was identified as Pohlia andalusica. Pohlia annotina has been recorded in Estonia from a new locality rather recently (Leis \& Kannukene, 2007). S. strictum was presented in the previous list as a frequent species in Estonia, but is now eliminated from the list since in the 1994 list S. apocarpum subsp. gracile was considered

Table 1. The comparison of numbers of different taxonomical units in the two lists of Estonian bryophytes

\begin{tabular}{lllllll}
\hline & \multicolumn{2}{l}{$\begin{array}{l}\text { Hornworts } \\
\text { Anthocerotophyta }\end{array}$} & \multicolumn{2}{l}{ Liverworts Marchantiophyta } & \multicolumn{2}{l}{$\begin{array}{l}\text { Mosses } \\
\text { Bryophyta }\end{array}$} \\
\hline Class & 1994 & 2015 & 1994 & 2015 & 1994 & 2015 \\
Order & 1 & 1 & 1 & 6 & 1 & 5 \\
Family & 1 & 2 & 3 & 18 & 16 & 20 \\
Genus & 1 & 2 & 25 & 32 & 37 & 54 \\
Species & 2 & 2 & 42 & 59 & 117 & 159 \\
subsp./var & 2 & 2 & 112 & 127 & 396 & 465 \\
\hline
\end{tabular}


to be a synonym of $S$. strictum due to misinterpretation. All specimens of that species belong to $S$. trichodon var. trichodon according to the revision of Schistidium apocarpum compex by Blom (1996).

Here we present locality data for two new species for Estonia:

1) Grimmia longirostris, loc. 1: Lääne-Viru Co., Vihula District, on a stone near sea coast behind the building of the Käsmu Sea Museum, 03 August 2008, leg/det. M.Leis (TAA); loc. 2; Harju Co., Kuusalu District, on a stone in open area, near bus station of Kolga-Aabla, 13 August 2008, leg./det. M.Leis (TAA).

2) Didymodon sicculus, loc.: Saaremaa Co., Muhu Island, Koguva limestone quarry, on a limestone, 07 July 2014, leg. E.Oja; 25 June 2015, det J.Kucera.

Only one species is known in Estonia by literature data, all other taxa have voucher specimens at different herbaria. In the review of N. Malta (1930) the occurrence of Dicranella rufescens in Estonia was noticed without indicating the herbarium specimens.

All voucher specimens are registered in eBiodiversity - a database for the taxa found in Estonia (http://elurikkus.ut.ee/). Two species have voucher specimens outside Estonia, both in Swedish Museum of Natural History (S). The record of Tortula lindbergii as a new species for Estonia was published in 1990 sub nom. Pottia lanceolata (Hedw.) C.Müll. (Hedenäs, 1990) and the specimen holds registration number B216405 in S. Jungermannia subulata was identified for Estonia by J. Váňa (1973) from Mikutowicz' exciccatae collection of Bryotheca Baltica, no 459 (S; reg. no B216672). In TU we have also the Mikutowicz' exciccatae collection, but under specimen no 459 only Cephalozia pleniceps was found.
Through numerous inventories of Estonian bryoflora our knowledge about the frequencies of species has improved. Altogether 242 species $(40 \%)$ are frequent in Estonia, 173 species $(29 \%)$ are rare, and 161 species $(27 \%)$ are sporadically distributed. For twenty species we do not have any recent data, and their presence in Estonia is doubtful, one of these is reported for Estonia only by literature source. Some species estimated as frequent earlier have scarce recent data and are now evaluated as sporadically distributed.

Altogether 369 species, $62 \%$ of the whole Estonian bryoflora, has been evaluated against IUCN criteria (categories (Red Data List 2008: http:/ / elurikkus.ut.ee/prmt.php?lang=eng). 155 species of these are considered as species with no risk, a little less (129 species) belong to the three threat categories (Table 2). For ten species, that have been considered to be extinct from Estonian bryoflora, new records have been registered. For example, Loeskypnum badium was found for the first time in 1904, and in 2012 the second locality was registered; Kiaeria blytti had only old literature data, but in 2009 a new locality was found on Mohni Island. Five spescies had insuffient data for evaluating them against IUCN threat categories. For example Sphagnum auriculatum had only two old records, and was evaluated as data deficient (DD) according to IUCN categories in 2008. One of these localities in a paludified forest in North-Estonia has been degraded due to drainage (Vellak et al., 2013), but two new records from small lakes in South-Estonia were found in 2014 and the status of $S$. auriculatum could now be evaluated as vulnerable.

Since red list does not have legal power for protection of species, 24 bryophyte species were taken under state protection in 1994 for the first

Table 2. Share of evaluated species in different IUCN categories (Red Data List 2008: http:// elurikkus.ut.ee/prmt.php?lang=eng)

\begin{tabular}{llllllll}
\hline \multicolumn{7}{c}{} & \multicolumn{7}{c}{ IUCN category } \\
\hline Taxon group & RE & CR & EN & VU & NT & DD & LC \\
\hline Hornworts & - & - & - & 1 & - & - & - \\
Liverworts & 5 & 2 & 2 & 25 & 14 & 1 & 36 \\
Mosses & 21 & 9 & 18 & 72 & 41 & 4 & 118 \\
\hline Total & $\mathbf{2 6}$ & $\mathbf{1 1}$ & $\mathbf{2 0}$ & $\mathbf{9 8}$ & $\mathbf{5 5}$ & $\mathbf{5}$ & $\mathbf{1 5 4}$ \\
\hline
\end{tabular}


time (Riigi Teataja, 1994). In 2004 and in 2014 several corrections have been made, and the latest version includes altogether 45 bryophyte species in three protection categories (Riigi Teataja, 2014a, b). One species, Rhynchostegium murale was eliminated from the present list since the state of the species has improved due to several newly discovered localities.

Decision for the IUCN categories at country level are made on the basis of species occurrence and state in particular region, therefore the renewal of county checklists is important for judgement of the status and protection need of species at larger regional levels. Efficient species conservation planning can be based also on the results obtained by monitoring. Since 1994, when the first list of protected species was compiled, also the bryophyte monitoring started in Estonia. At present 26 species are included in the state monitoring program, five species of these belong to the EU Habitat Directive list (Vellak \& Ingerpuu, 2012).

The present checklist includes altogether 76 species that have been discovered by more thorough investigation during last twenty years. It is possible that most of these species have always been part of Estonian bryoflora (Rhytidiadelphus loreus, Sphagnum pulchrum etc.), but some of them are newcomers, e.g. Campylopus introflexus, that is an invasive species in Europe (Hassel \& Södersröm, 2005). On the other hand we do not have recent localities for 19 species and they may be lost from our bryoflora, also some species have decreased significantly their distribution. The reason for such losses can be climate change (Splachnum spp., Meesia longiseta), but the main cause is still human impact. The reduction and damage of habitats threatens especially sensitive or rare species. Altogether 92 species occurring in Estonia are evaluated as candidate species for the red list of European bryophytes (Hodgetts, 2015), nine species of them have no recent records in Estonia. Here elaborate and efficient conservation methods can improve the state of threatened species, since the decline of such species distribution range starts long before the results are visible at larger global scale (Hallingbäck, 2002).

\section{CHECKLIST OF ESTONIAN BRYOPHYTES}

List of Estonian bryophytes with their distribution frequency (Freq), IUCN category in Red List of Estonia (ERL 2008), State Protection Category in 2014 (LK 2014), and indication of proofing source (voucher). Abbreviations: Frequency classes: $r$ - rare (1-7 documented findings); $\mathrm{p}$ - sporadically (8-30 localities); fq - frequent, (31 or more localities); ? - doubtful (no records after 1950). Herbaria: S - Swedish Museum of Natural History; TAA - Herbarium of the Estonian University of Life Sciences; TALL - Herbarium of the Tallinn Botanical Garden; TAM - Herbarium of the Estonian Natural History Museum; TU - Herbarium of the Natural History Museum of the University of Tartu.

\begin{tabular}{lc}
\hline Accepted name & Freq ERL LK 2014 Voucher \\
2008 & \\
\hline
\end{tabular}

DIVISON ANTHOCEROTOPHYTA Rothm. ex Stotler \& Crand.-Stotl.

Anthoceros agrestis Paton

Phaeoceros carolinianus (Michx.) Prosk.

TU169164

DIVISION MARCHANTIOPHYTA Stotler \& Crand.-Stotl.

Aneura pinguis (L.) Dumort.

Barbilophozia barbata (Schmidel ex Schreb.) Loeske

p TU169164

r VU TAA5004627

Barbilophozia hatcheri (A.Evans) Loeske

Barbilophozia lycopodioides (Wallr.) Loeske

Barbilophozia sudetica (Nees ex Huebener) L.Söderstr., De Roo \& Hedd.

Bazzania trilobata (L.) Gray

Blasia pusilla L.

Blepharostoma trichophyllum (L.) Dumort.

Calypogeia azurea Stotler \& Crotz

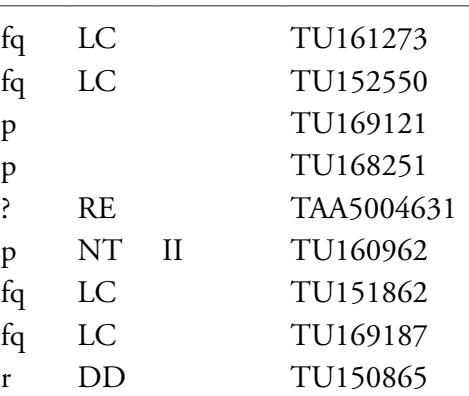

TU161273

TU169121

TU168251

TU160962

TU169187

TU150865 
Calypogeia integristipula Steph.

Calypogeia muelleriana (Schiffn.) Müll.Frib.

Calypogeia neesiana (C.Massal \& Carestia) Müll.Frib.

Calypogeia sphagnicola (Arnell \& J.Perss.) Warnst. \& Loeske

Calypogeia suecica (Arnell \& J.Perss.) Müll.Frib.

Cephalozia bicuspidata (L.) Dumort.

var. bicuspidata

var. lammersiana (Huebener) Breidl.

Cephalozia pleniceps (Austin) Lindb.

Cephaloziella divaricata (Sm.) Schiffn.

Cephaloziella elachista (J.B.Jack) Schiffn.

Cephaloziella hampeana (Nees) Schiffn.

Cephaloziella integerrima (Lindb.) Warnst.

Cephaloziella rubella (Nees) Warnst.

Cephaloziella spinigera (Lindb.) Warnst.

Chiloscyphus pallescens (Ehrh. ex Hoffm.) Dumort.

var. pallescens

var. fragilis (A.Roth) Müll.Frib.

Chiloscyphus polyanthos (L.) Corda

Clevea hyalina (Sommerf.) Lindb.

Conocephalum conicum (L.) Dumort.

Conocephalum salebrosum Szweykowski et al.

Crossocalyx hellerianus (Nees ex Lindenb.) Meyl.

Endogemma caespiticia (Lindenb.) Konstant., Vilnet \& A.V.Troitsky

Fossombronia foveolata Lindb.

Fossombronia wondraczekii (Corda) Dumort.

Frullania dilatata (L.) Dumort.

Frullania tamarisci (L.) Dumort.

Fuscocephaloziopsis catenulata (Huebener) Váňa \& L.Söderstr.

Fuscocephaloziopsis connivens (Dicks.) Váňa \& L.Söderstr.

Fuscocephaloziopsis loitlesbergeri (Schiffn.) Váňa \& L.Söderstr.

Fuscocephaloziopsis lunulifolia (Dumort.) Váňa \& L.Söderstr.

Geocalyx graveolens (Schrad.) Nees

Gymnocolea inflata (Huds.) Dumort.

Harpantus flotovianus (Nees) Nees

Harpantus scutatus (F.Weber \& D.Mohr) Spruce

Heterogemma laxa (Lindb.) Konstant. \& Vilnet

Isopaches bicrenatus (Schmidel) H. Buch

Jungermannia atrovirens Dumort.

Kurzia pauciflora (Dicks.) Grolle

Lejeunea cavifolia (Ehrh.) Lindb.

Lepidozia reptans (L.) Dumort.

Liochlaena lanceolata Nees

Liochlaena subulata (A.Evans) Schljakov

Lophocolea bidentata (L.) Dumort.

Lophocolea heterophylla (Schrad.) Dumort.

Lophocolea minor Nees $\mathrm{fq}$

$\mathrm{fq}$

$\mathrm{p}$

$\mathrm{p}$

$\mathrm{fq}$

LC

TU161023

TAA5000405

TU152559

TU150818

TU150978

TU167314

TU151842

TU168250

TU167210

TU152409

TAA5004619

fq LC

? $\mathrm{RE}$

$\mathrm{p}$

$\mathrm{p}$

fq LC

p NT

r VU

r NT

fq

$r$

$r$

f

$p$

fq

$\mathrm{p}$

$\mathrm{p}$

$\mathrm{r}$

r

$\mathrm{r}$

$\mathrm{p}$

$r$

fq LC

$\mathrm{p}$

fq LC

$\mathrm{p}$

? $\mathrm{RE}$

fq

fq LC

fq
TU160486

TU150201

TU168284

TU161125

TU169124

TAA5004622

TU160492

TU169163

TU151845

TU152599

TU160445

TU167330

TAA5004618

TU168643

TU167060

TU168058

TAA5004620

TAA5004621

TU167852

TU167317

TU160511

TU160521

TU151867

TU169186

TU150918

S;reg.no.B216672

TU151848

TU161017

TU160539 
Lophozia ascendens (Warnst.) R.M.Schust.

$\begin{array}{lll}\mathrm{r} & \mathrm{VU} & \text { TU161024 } \\ \mathrm{fq} & & \text { TU160568 } \\ \mathrm{r} & & \text { TU161368 } \\ \mathrm{fq} & & \text { TU160969 } \\ \mathrm{p} & & \text { TU160557 } \\ \mathrm{p} & & \text { TU151902 } \\ \mathrm{r} & \mathrm{VU} & \text { TU167828 } \\ \mathrm{r} & \text { VU } & \text { TU160588 } \\ \mathrm{fq} & \text { LC } & \end{array}$

Lophozia guttulata (Lindb. \& Arnell) A.Evans

TU169327

Lophozia silvicola $\mathrm{H}$.Buch

Lophozia ventricosa (Dicks.) Dumort.

Lophoziopsis excisa (Dicks.) Konstant. \& Vilnet

TAA5004625

subsp. montivagans Bischl. \& Boisselier-Dubayle

TU168420

subsp. ruderalis Bischl. \& Boisselier-Dubayle

TU160972

Mesoptychia badensis (Gottsche) L.Söderstr. \& Váňa

Mesoptychia bantriensis (Hook.) L.Söderstr. \& Váňa

TU161286

Mesoptychia heterocolpos (Thed.) L.Söderstr. \& Váňa

p NT

TU152414

Mesoptychia rutheana (Limpr.) L.Söderstr. \& Váňa

Metzgeria conjugata Lindb.

Metzgeria furcata (L.) Dumort.

Moerckia hibernica (Hook.) Gottsche

Mylia anomala (Hook.) Gray

Nardia geoscyphus (De Not.) Lindb.

Nardia insecta Lindb.

Neoorthocaulis attenuatus (Mart.) L.Söderstr., De Roo, Hedd. \& Mart.

r VU

p NT

TU168413

TU169268

TU169123

TU168520

TU151852

TAA5004626

TU167318

TU167287

Neoorthocaulis floerkei (F.Weber \& D.Mohr) L.Söderstr., De Roo \& Hedd. ～? $\quad$ EN

Nowellia curvifolia (Dicks.) Mitt.

Obtusifolium obtusum (Lindb.) S.W.Arnell

Odontoschisma denudatum (Mart.) Dumort.

Odontoschisma fluitans (Nees) L. Söderstr. \& Váňa

fq LC

TAM B18:58

TU169418

TU168077

TU160958

TU151844

Oleolophozia perssonii (H. Buch \& S.W.Arnell) L.Söderstr., De Roo \& Hedd.

Pellia endiviifolia (Dicks.) Dumort.

Pellia epiphylla (L.) Corda

fq LC

r VU

$\mathrm{p}$

fq $\quad$ LC

TU167878

Pellia neesiana (Gottsche) Limpr.

Plagiochila asplenioides (L.) Dumort.

Plagiochila porelloides (Torrey ex Nees) Lindenb.

Porella cordaeana (Huebener) Moore

Porella platyphylla (L.) Pfeiff.

Preissia quadrata (Scop.) Nees

Ptilidium ciliare (L.) Hampe

Ptilidium pulcherrimum (Weber) Vain.

Radula complanata (L.) Dumort.

Reboulia hemisphaerica (L.) Raddi

Riccardia chamaedryfolia (With.) Grolle

Riccardia incurvata Lindb.

$r \quad$ VU II

TU162053

TU162128

fq $\quad$ LC

TU168064

TU168639

fq LC

TU150881

fq $\quad$ LC

TU169136

p NT III

TU167874

p NT III

TU168588

fq LC

TU169407

TU169241

TU152747

TU167829

TU167329

TU150913

Riccardia latifrons (Lindb.) Lindb.

TU161279

Riccardia multifida (L.) Gray

Riccardia palmata (Hedw.) Carruth.

TU167785

TU160647

Riccia beyrichiana Hampe ex Lehm.

TU150820 
Riccia bifurca Hoffm.

fq LC

TU160651

Riccia cavernosa Hoffm.

TU160653

Riccia ciliata Hoffm.

TAA5004628

TU152597

TU160657

Riccia fluitans $\mathrm{L}$.

Riccia glauca L.

Riccia sorocarpa Bisch.

Riccia warnstorfi Limpr. ex Warnst.

Ricciocarpos natans (L.) Corda

Scapania apiculata Spruce

Scapania calcicola (Arnell \& J.Perss.) Ingham

Scapania curta (Mart.) Dumort.

Scapania gymnostomophila Kaal.

Scapania irrigua (Nees) Nees

Scapania lingulata $\mathrm{H}$.Buch

Scapania mucronata H.Buch

Scapania nemorea (L.) Crolle

Scapania paludicola Loeske \& Müll.Frib.

Scapania umbrosa (Schrad.) Dumort.

Scapania undulata (L.) Dumort.

Schistochilopsis incisa (Schrad.) Konstant.

Schistochilopsis opacifolia (Culm.) Konstant.

Schljakovia kunzeana (Huebener) Konstant. \& Vilnet

$\mathrm{p}$

$\mathrm{p}$

fq LC

r VU

p LC

p NT III

p NT

$\mathrm{p}$

$\mathrm{r}$

$\mathrm{fq}$

fa

$\mathrm{p}$

$\mathrm{p}$

r

$\mathrm{p}$

$\mathrm{r}$

$r$

$\mathrm{p}$

?

Solenostoma confertissimum (Nees) Schljakov

Solenostoma gracillimum (Sm.) R.M.Schust.

Solenostoma hyalinum (Lyell) Mitt.

Solenostoma sphaerocarpum (Hook.) Steph.

Sphenolobus minutus (Schreb.) Berggr.

Syzygiella autumnalis (DC.) K.Feldberg, Váňa, Hentschel \& J.Heinrichs

Trichocolea tomentella (Ehrh.) Dumort.

Tritomaria exsectiformis (Breidl.) Loeske

Tritomaria quinquedentata (Huds.) H.Buch

\section{DIVISION BRYOPHYTA Schimp.}

Abietinella abietina (Hedw.) M.Fleisch.

Alleniella complanata (Hedw.) S.Olsson, Enroth \& D.Quandt

Aloina rigida (Hedw.) Limpr.

Amblyodon dealbatus (Hedw.) P.Beauv.

Amblystegium serpens (Hedw.) Schimp.

Andreaea rupestris $\mathrm{Hedw}$.

Anomodon attenuatus (Hedw.) Huebener

Anomodon longifolius (Schleich. ex Brid.) Hartm.

Anomodon rugelii (Müll.Hal.) Keissl.

Anomodon viticulosus (Hedw.) Hook. \& Taylor

Antitrichia curtipendula (Hedw.) Brid.

Aplodon wormiskioldii (Hornem.) R.Br.

Atrichum angustatum (Brid.) Bruch \& Schimp.

Atrichum crispum (James) Sull.

Atrichum flavisetum Mitt.

$\mathrm{p} \quad \mathrm{NT}$
TU160658

TU160659

TU150906

TU167884

TU160966

TU168349

TU160664

TU150967

TU160668

TU161355

TU162044

TU168428

TAA5004629

TU150761

TU167839

TAA5004630

TU161295

TAA5004623

TU152277

TU160515

TAA5004624

TU161118

TU167840

TU169180

TU160681

TU161013
TAA5000005

TU170010

TAM B803:1

TAA5004602

TU151886

TU161121

TAA5000177

TAA5000186

TAA5000210

TAA5000213

TU161309

TU158429

TAA5000227

TU157121

TU151085 
Atrichum tenellum (Röhl.) Bruch \& Schimp.

$\mathrm{p}$

Atrichum undulatum (Hedw.) P.Beauv.

Aulacomnium androgynum (Hedw.) Schwägr.

Aulacomnium palustre (Hedw.) Schwägr.

Barbula convoluta Hedw.

Barbula unguiculata $\mathrm{Hedw}$.

Bartramia ithyphylla Brid.

Bartramia pomiformis Hedw.

Brachytheciastrum velutinum (Hedw.) Ignatov \& Huttunen

Brachythecium albicans (Hedw.) Schimp.

Brachythecium campestre (Müll.Hal.) Schimp.

Brachythecium erythrorrhizon Schimp.

Brachythecium glareosum (Bruch ex Spruce) Schimp.

Brachythecium mildeanum (Schimp.) Schimp.

Brachythecium rivulare Schimp.

Brachythecium rutabulum (Hedw.) Schimp.

Brachythecium salebrosum (Hoffm. ex F.Weber \& D.Mohr) Schimp., nom.cons.

Brachythecium tommasinii (Sendtn. ex Boulay) Ignatov \& Huttunen

Brachythecium turgidum (Hartm.) Kindb.

Breidleria pratensis (W.D.J. Koch ex Spruce) Loeske

Bryoerythrophyllum recurvirostrum (Hedw.) P.C.Chen

Bryum argenteum $\mathrm{Hedw}$.

Bryum blindii Bruch \& Schimp.

Bryum calophyllum R.Br.

Bryum dichotomum Hedw.

Bryum elegans Nees

Bryum funckii Schwägr.

Bryum intermedium (Brid.) Blandow

Bryum klinggraeffi Schimp.

Bryum knowltonii Barnes

Bryum kunzei Hornsch.

Bryum marratii Hook.f. \& Wilson

Bryum radiculosum Brid.

Bryum salinum I.Hagen ex Limpr.

Bryum subapiculatum Hampe

Bryum turbinatum (Hedw.) Turner

Bryum warneum (Röhl.) Brid.

Bryum weigelii Spreng.

Buxbaumia aphylla Hedw.

Buxbaumia viridis (Moug. ex Lam. \& DC.) Brid. ex Moug. \& Nestl.

Callicladium haldanianum (Grev.) H.A.Crum

Calliergon cordifolium (Hedw.) Kindb.

Calliergon giganteum (Schimp.) Kindb.

Calliergon megalophyllum Mikut.

Calliergon richardsonii (Mitt.) Kindb.

Calliergonella cuspidata (Hedw.) Loeske

Calliergonella lindbergii (Mitt.) Hedenäs $\mathrm{fq}$

$\mathrm{fq}$

fq $\quad$ LC

fq $\quad$ LC

$\mathrm{fq}$$$
\text { ? }
$$

r

fq LC

fq LC

$\mathrm{p} \quad \mathrm{NT}$

$\mathrm{fq}$

fq

fq

$\mathrm{fq}$

$\mathrm{fq}$

$\mathrm{r}$

$\mathrm{p}$

$\mathrm{p}$

$\mathrm{f}$

fq

$\mathrm{r}$

r

$\mathrm{r}$

$r$

$\mathrm{p}$

$\mathrm{r}$

$\mathrm{p}$

$r$

$r$

$\mathrm{r}$

r

$r$

r

r

$r$

r

$r$

$\mathrm{p}$

$\mathrm{r}$

r

$\mathrm{p}$

$\mathrm{p}$

$\mathrm{fq}$

fq LC

fq LC

r VU

p NT

fq LC

fq
TU157123

TU168095

TAM0069986

TAM B866:125

TAM B837:250

TAM B824:85

TU151433

TU167030

TAM0070205

TAM0056236

TAM B796:27

TAM B772:19

TAM B845:158

TAM B794:9

TAM B837:153

TAM B821:112

TAM B868:259

TU167010

TAM B808:11

TU152479

TAM B847:62

TU158545

TAA5001159

TU167802

TU151916

TU161126

TAA5001210

TU167385

TAM B868:206

TAM B771:57

TAM B783:133

TU168090

TU152704

TAA5004603

TAA5001251

TAM B767:40

TU168283

TU167357

TU169408

TU168623

TU167065

TU169388

TU168664

TAA5001304

TU150953

TU151912

TU168233 
Campyliadelphus chrysophyllus (Brid.) R.S.Chopra

Campyliadelphus elodes (Lindb.) Kanda

Campylidium calcareum (Crundw. \& Nyholm) Ochyra

Campylidium sommerfeltii (Myrin) Ochyra

Campylium protensum (Brid.) Kindb.

Campylium stellatum (Hedw.) Lange \& C.E.O.Jensen

Campylophyllum halleri (Hedw.) M.Fleisch.

Campylopus fragilis (Brid.) Bruch \& Schimp.

Campylopus introflexus (Hedw.) Brid.

Campylopus pyriformis (Schultz) Brid.

Catoscopium nigritum (Hedw.) Brid.

Ceratodon conicus (Hampe) Lindb.

Ceratodon purpureus (Hedw.) Brid.

Cinclidium stygium Sw.

Cirriphyllum piliferum (Hedw.) Grout

Climacium dendroides (Hedw.) F.Weber \& D.Mohr

Conardia compacta (Drumm. ex Müll.Hal.) H.Rob.

Cratoneuron filicinum (Hedw.) Spruce

Ctenidium molluscum (Hedw.) Mitt.

Cynodontium bruntonii (Sm.) Bruch \& Schimp.

Cynodontium polycarpon (Hedw.) Schimp.

Cynodontium strumiferum (Hedw.) Lindb.

Dichelyma capillaceum (L. ex Dicks.) Myrin

Dichelyma falcatum (Hedw.) Myrin

Dichodontium pellucidum (Hedw.) Schimp.

Dicranella cerviculata (Hedw.) Schimp.

Dicranella crispa (Hedw.) Schimp.

Dicranella heteromalla (Hedw.) Schimp.

Dicranella humilis R. Ruthe

Dicranella rufescens (Dicks.) Schimp.

Dicranella schreberiana (Hedw.) Dixon

Dicranella subulata (Hedw.) Schimp.

Dicranella varia (Hedw.) Schimp.

Dicranoweisia cirrata (Hedw.) Lindb.

Dicranum bonjeanii De Not.

Dicranum brevifolium (Lindb.) Lindb.

Dicranum drummondii Müll.Hal.

Dicranum flagellare Hedw.

Dicranum flexicaule Brid.

Dicranum fuscescens Sm.

Dicranum leioneuron Kindb.

Dicranum majus Sm.

Dicranum montanum Hedw.

Dicranum polysetum Sw. ex anon.

Dicranum scoparium Hedw.

Dicranum spurium Hedw.

Dicranum undulatum Schrad. ex Brid. fq

fq

$\mathrm{fq}$

$\mathrm{fq}$

$\mathrm{fq}$

$\mathrm{fq}$

$$
r
$$

r

$r$

r$$
r
$$

fq

$\mathrm{fq}$

$\mathrm{f}$

fo$$
r
$$

fq

$\mathrm{fq}$

$r$

$r$

$\mathrm{p}$

$\mathrm{r}$

r

\section{$r$}

$\mathrm{p}$

fq

$\mathrm{p}$

fq

$\mathrm{r}$

TU168232

TU161335

TU160970

TU152535

TU161272

TU158019

TU162015

TU151120

TAA5001408

TU157690

TU160975

TU157825

TAA5001430

TU169177

TAM0069888

TAA5001469

TU167296

TU161250

TU152458

TAA5000474

TAA5000475

TAA5000485

TU151820

TU169426

TAA5000519

TAA5001506

TAA5000533

TAA5001541

TAA5004582

Malta 1930

TAA5000544

TAA5000559

TAA5001546

TAA5000561

TAA5001595

TAA5000564

TAA5004583

TAA5001606

TAA5000590

TAA5001614

TAA500061 1

TAA5001641

TAA5001668

TAA5001685

TAA5001695

TAA5000639

TAA5001560 
Dicranum viride (Sull. \& Lesq.) Lindb.

Didymodon fallax (Hedw.) R.H.Zander

Didymodon ferrugineus (Schimp. ex Besch.) M.O.Hill

Didymodon insulanus (De Not.) M.O.Hill

Didymodon rigidulus $\mathrm{Hedw}$.

Didymodon sicculus Cano, Ros, Carcía-Zamora \& J.Guerra

Didymodon tophaceus (Brid.) Lisa

Didymodon vinealis (Brid.) R.H.Zander

Diphyscium foliosum (Hedw.) D.Mohr

Discelium nudum (Dicks.) Brid.

Distichium capillaceum (Hedw.) Bruch \& Schimp.

Distichium inclinatum (Hedw.) Bruch \& Schimp.

Ditrichum flexicaule (Schwägr.) Hampe

Ditrichum lineare (Sw.) Lindb.

Ditrichum pallidum (Hedw.) Hampe

Ditrichum pusillum (Hedw.) Hampe

Drepanocladus aduncus (Hedw.) Warnst.

Drepanocladus longifolius (Mitt.) Paris

Drepanocladus lycopodioides (Brid.) Warnst.

Drepanocladus polygamus (Schimp.) Hedenäs

Drepanocladus sendtneri (Schimp. ex H.Müll.) Warnst.

Drepanocladus sordidus (Müll.Hal.) Hedenäs

Drepanocladus trifarius (F. Weber \& D.Mohr) Loeske

Drepanocladus turgescens (T.Jensen) Loeske

Encalypta ciliata Hedw.

Encalypta mutica I.Hagen

Encalypta rhabtocarpa Schwägr.

Encalypta streptocarpa $\mathrm{Hedw}$.

Encalypta vulgaris Hedw.

Ephemerum serratum (Hedw.) Hampe

Eucladium verticillatum (With.) Bruch \& Schimp.

Eurhynchiastrum pulchellum (Hedw.) Ignatov \& Huttunen

var. pulchellum

var. praecox (Hedw.) Ochyra \& Żarnowiec

Eurhynchium angustirete (Broth.) T.J.Kop.

Eurhynchium striatum (Hedw.) Schimp.

Exsertotheca crispa (Hedw.) S.Olsson, Enroth \& D.Quandt

Fissidens adianthoides Hedw.

Fissidens arnoldii R. Ruthe

Fissidens bryoides Hedw.

Fissidens dubius P.Beauv.

Fissidens exilis Hedw.

Fissidens fontanus (Bach.Pyl.) Steud.

Fissidens gracilifolius Brugg.-Nann. \& Nyholm

Fissidens osmundoides Hedw.

Fissidens pusillus (Wilson) Milde

Fissidens taxifolius Hedw.

$\begin{array}{llll}\mathrm{p} & \mathrm{NT} & \mathrm{II} & \text { TAA5000655 } \\ \mathrm{fq} & & \text { TAM0056317 } \\ \mathrm{p} & & \text { TAM B744:39 } \\ \mathrm{p} & \mathrm{VU} & \text { TAM B737:74 } \\ \mathrm{fq} & \mathrm{LC} & \text { TAM0056280 } \\ \mathrm{r} & & \text { TU169462 } \\ \mathrm{p} & \mathrm{NT} & \text { TAM0056437 } \\ \mathrm{r} & \mathrm{VU} & \text { TAM B812:20 } \\ \mathrm{r} & \mathrm{VU} & \text { TAA5004594 } \\ \mathrm{r} & \mathrm{RE} & \text { TAM B853:70 } \\ \mathrm{fq} & \mathrm{LC} & \text { TAA5001769 } \\ \mathrm{p} & \mathrm{NT} & \text { TAA5000667 } \\ \mathrm{fq} & \text { LC } & \text { TAA5001778 } \\ \mathrm{r} & \mathrm{VU} & \text { TAA5000703 } \\ \mathrm{r} & \mathrm{VU} & \text { TAA5000704 } \\ \mathrm{r} & \mathrm{VU} & \text { TAA5000705 } \\ \mathrm{fq} & \text { LC } & \text { TU161306 } \\ \mathrm{p} & \mathrm{VU} & \text { TU168278 } \\ \mathrm{p} & & \text { TU159257 } \\ \mathrm{fq} & & \text { TU160974 } \\ \mathrm{fq} & & \text { TU161008 } \\ \mathrm{p} & & \text { TU168001 } \\ \mathrm{p} & \mathrm{NT} & \text { TU168663 } \\ \mathrm{fq} & & \text { TU167806 } \\ \mathrm{r} & \mathrm{EN} & \text { TAA5004595 } \\ \mathrm{r} & \mathrm{VU} & \mathrm{II} & \text { TAM B764:94 } \\ \mathrm{fq} & & \text { TAM0056313 } \\ \mathrm{fq} & \text { LC } & \text { TAM0070277 } \\ \mathrm{fq} & & \text { TAM B823:80 } \\ \mathrm{r} & \mathrm{VU} & \text { TAM B813:12 } \\ \mathrm{r} & \mathrm{RE} & \text { TAA5004592 } \\ \mathrm{fq} & & \\ \mathrm{f} & & \mathrm{TAM} \mathrm{B874:353}\end{array}$

TAM B874:353

TU152212

TAM B876:234

TAM B837:38

TU152490

TAA5001960

TAA5000706

TAA5000708

TAA5004584

TU152631

TAA5000726

TAA5000729

TAA5001998

TAA5004590

TAA5002031 
Fontinalis antipyretica Hedw.

fq LC

subsp. antipyretica

TU151184

subsp. gracilis (Lindb.) Kindb.

TU170102

Fontinalis dalecarlica Schimp.

TAA5004607

Fontinalis hypnoides C.Hartm.

Fontinalis squamosa Hedw.

Funaria hygrometrica Hedw.

Grimmia anomala Hampe ex Schimp.

Grimmia crinita Brid.

Grimmia hartmanii Schimp.

Grimmia laevigata (Brid.) Brid.

Grimmia longirostris Hook.

Grimmia muehlenbeckii Schimp.

Grimmia ovalis (Hedw.) Lindb.

Grimmia pulvinata (Hedw.) Sm.

Grimmia trichophylla Grev.

Gymnostomum aeruginosum Sm.

Gymnostomum calcareum Nees \& Hornsch.

Gyroweisia tenuis (Hedw.) Schimp.

Hamatocaulis lapponicus (Norrl.) Hedenäs

Hamatocaulis vernicosus (Mitt.) Hedenäs

Hedwigia ciliata (Hedw.) P.Beauv.

var. ciliata

r VU

p VU

TU167049

TAA5004608

fq LC TAA5002090

TAM B872:60

TALL D009254

TAM B863:330

TAM B813:14

TAA5004597

TAM B829:318

TAM0070032

TAM B821:202

TAM B803:222

TAM B803:226

TAM B837:143

TAM B853:62

var. leucophaea Bruch \& Schimp.

Hedwigia stellata Hedenäs

Helodium blandowii (F.Weber \& D.Mohr) Warnst.

NT

TAM B853:62

NT

TU167562

fq NT III

TU168417

$\mathrm{fq}$

TU169352

TAM B837:105

TAA5002178

TAA5002193

TAA5000503

Hennediella heimii (Hedw.) R.H.Zander

Herzogiella seligeri (Brid.) Z.Iwats.

Herzogiella striatella (Brid.) Z.Iwats.

Herzogiella turfacea (Lindb.) Z.Iwats.

Homalia trichomanoides (Hedw.) Brid.

Homalothecium lutescens (Hedw.) H.Rob.

Homalothecium sericeum (Hedw.) Schimp.

Homomallium incurvatum (Schrad. ex Brid.) Loeske

Hygroamblystegium fluviatile (Hedw.) Loeske

Hygroamblystegium humile (P.Beauv.) Vanderp., Goffinet \& Hedenäs

TU168642

TAA5002216

TU160038

$r \quad$ VU

TU158880

fq LC

TAM B866:85

TAM B851:69

TU160081

TU158039

Hygroamblystegium tenax (Hedw.) Jenn.

TAA5000018

Hygroamblystegium varium (Hedw.) Mönk.

TU167302

TU152529

Hygrohypnum luridum (Hedw.) Jenn.

Hylocomiastrum umbratum (Hedw.) M.Fleisch.

TU161005

Hylocomium splendens (Hedw.) Schimp.

Hymenostylium recurvirostrum (Hedw.) Dixon

Hypnum cupressiforme Hedw.

var. cupressiforme

var. filiforme Brid.

var. Lacunosum Brid.

TU161297

TU168686

TAM0056368

fq LC

TU152715

TU161458

TU151949

Hypnum fertile Sendtn.

? $\quad \mathrm{RE}$

TU171737 
Hypnum pallescens (Hedw.) P.Beauv.

Isopterygiopsis pulchella (Hedw.) Z.Iwats.

TU168472

Isothecium alopecuroides (Lam. ex Dubois) Isov.

Isothecium myosuroides Brid.

Kiaeria blyttii (Bruch \& Schimp.) Broth.

Kindbergia praelonga (Hedw.) Ochyra

Leptobryum pyriforme (Hedw.) Wilson

Leptodictyum riparium (Hedw.) Warnst.

Leskea polycarpa Hedw.

Leucobryum glaucum (Hedw.) Ångstr.

Leucodon sciuroides (Hedw.) Schwägr.

Loeskypnum badium (Hartm.) H.K.G.Paul

Meesia longiseta Hedw.

Meesia triquetra (L. ex Jolycl.) Ångstr.

Meesia uliginosa Hedw.

Microbryum davallianum (Sm.) R.H.Zander

Microbryum floerkeanum (F.Weber \& D.Mohr) Schimp.

Microbryum starckeanum (Hedw.) R.H.Zander

Mnium hornum Hedw.

Mnium marginatum (Dicks.) P.Beauv.

r EN

TAM B844:212

$\mathrm{p}$

$\mathrm{p} \quad \mathrm{NT}$

TU159495

TU160957

$r \quad \mathrm{RE}$

fq

fq $\quad$ LC

$\mathrm{fq} \quad \mathrm{LC}$

TAA5000776

TAM B824:7

TU151978

TU158043

fq LC III

TU167294

Mnium stellare Hedw.

Myrinia pulvinata (Wahlenb.) Schimp.

fq LC

TAA5002428

TAM B874:88

TU160948

TAA5004611

TU168427

TAA5004612

TAA5000807

TAA5000809

TALL D010208

TU162046

TU168282

TU167830

Myurella julacea (Schwägr.) Schimp.

Neckera pennata Hedw.

Nyholmiella gymnostoma (Bruch ex Brid.) Holmen \& E. Warncke

Nyholmiella obtusifolia (Brid.) Holmen \& E. Warncke

Oncophorus wahlenbergii Brid.

Orthotrichum affine Schrad. ex Brid.

Orthotrichum anomalum Hedw.

Orthotrichum cupulatum Hoffm. ex Brid.

Orthotrichum diaphanum Schrad. ex Brid.

Orthotrichum lyellii Hook. \& Taylor

Orthotrichum pallens Bruch ex Brid.

Orthotrichum patens Bruch ex Brid.

Orthotrichum pumilum Sw. ex anon.

Orthotrichum pylaisii Brid.

Orthotrichum rogeri Brid.

Orthotrichum rupestre Schleich. ex Schwägr.

Orthotrichum schimperi Hammar

Orthotrichum speciosum Nees

Orthotrichum stramineum Hornsch. ex Brid.

Oxyrrhynchium hians (Hedw.) Loeske

Oxystegus tenuirostris (Hook. \& Taylor) A.J.E.Sm.

Paludella squarrosa (Hedw.) Brid.

Palustriella commutata (Hedw.) Ochyra

Palustriella decipiens (De Not.) Ochyra

Palustriella falcata (Brid.) Hedenäs

TAM0011655

TU152467

TU151793

TAA5000846

TAA5002643

TU151503

TAA5000815

TAA5002613

TAA5002621

TAA5000836

TAA5000853

TAA5002656

TAA5000855

TAA5002665

TAA5000856

TAA5000858

TAA5002696

TAA5000862

TAA5002708

TU170014

TAM0069974

TU167402

TU168444

TU161282

TALL D012924

TU161281 
Paraleucobryum longifolium (Hedw.) Loeske

Pelekium minutulum (Hedw.) A.Touw

Philonotis caespitosa Jur.

Philonotis calcarea (Bruch \& Schimp.) Schimp.

Philonotis capillaris Lindb.

Philonotis fontana (Hedw.) Brid.

Physcomitrella patens (Hedw.) Bruch \& Schimp.

Physcomitrium eurystomum Sendtn.

Physcomitrium pyriforme (Hedw.) Bruch \& Schimp.

Plagiomnium affine (Blandow ex Funck) T.J.Kop.

Plagiomnium cuspidatum (Hedw.) T.J.Kop.

Plagiomnium elatum (Bruch \& Schimp.) T.J.Kop.

Plagiomnium ellipticum (Brid.) T.J.Kop.

Plagiomnium medium (Bruch \& Schimp.) T.J.Kop.

Plagiomnium rostratum (Schrad.) T.J.Kop.

Plagiomnium undulatum (Hedw.) T.J.Kop.

Plagiopus oederianus (Sw.) H.A.Crum \& L.E.Anderson

Plagiothecium cavifolium (Brid.) Z.Iwats.

Plagiothecium curvifolium Schlieph. ex Limpr.

Plagiothecium denticulatum (Hedw.) Schimp.

var. denticulatum

var. undulatum R.Ruthe ex Geh.

Plagiothecium laetum Schimp.

Plagiothecium latebricola Schimp.

Plagiothecium nemorale (Mitt.) A.Jaeger

Plagiothecium succulentum (Wilson) Lindb.

Plagiothecium undulatum (Hedw.) Schimp.

Platydictya jungermannioides (Brid.) H.A.Crum

Platygyrium repens (Brid.) Schimp.

Pleuridium subulatum (Hedw.) Rabenh.

Pleurozium schreberi (Willd. ex Brid.) Mitt.

Pogonatum aloides (Hedw.) P.Beauv.

Pogonatum dentatum (Menzies ex Brid.) Brid.

Pogonatum nanum (Hedw.) P.Beauv.

Pogonatum urnigerum (Hedw.) P.Beauv.

Pohlia andalusica (Höhn.) Broth.

Pohlia annotina (Hedw.) Lindb.

Pohlia bulbifera (Warnst.) Warnst.

Pohlia cruda (Hedw.) Lindb.

Pohlia elongata Hedw.

Pohlia lescuriana (Sull.) Ochi

Pohlia melanodon (Brid.) A.J.Shaw

Pohlia nutans (Hedw.) Lindb.

Pohlia proligera (Kindb.) Lindb. ex Broth.

Pohlia sphagnicola (Bruch \& Schimp.) Broth.

Pohlia wablenbergii (F.Weber \& D.Mohr) A.L.Andrews

\begin{tabular}{|c|c|c|}
\hline LC & & TAA5002753 \\
\hline RE & & TAM0081133 \\
\hline \multirow{4}{*}{\multicolumn{2}{|c|}{ NT }} & TU161274 \\
\hline & & TU161269 \\
\hline & & TAA50000871 \\
\hline & & TU158829 \\
\hline CR & & TAM B836:148 \\
\hline VU & & TAA5000873 \\
\hline LC & & TAA5002803 \\
\hline $\mathrm{LC}$ & & TU152498 \\
\hline $\mathrm{LC}$ & & TU168459 \\
\hline \multirow{4}{*}{\multicolumn{2}{|c|}{$\mathrm{LC}$}} & TU158650 \\
\hline & & TU158663 \\
\hline & & TU158634 \\
\hline & & TU167674 \\
\hline LC & & TU169266 \\
\hline \multirow[t]{2}{*}{ VU } & II & TU168447 \\
\hline & & TU168004 \\
\hline LC & & TU167726 \\
\hline
\end{tabular}

TU169269

TU167265

TU167988

TU167089

TU167857

TU167023

TU167660

TU168286

TU160941

TAA5000876

TU169405

TAA5004613

TU157011

TAA5004614

TU157020

TAA5004598

TU162056

TU158463

TU158473

TAA5003047

TU167135

TU151954

TU168463

TU161123

TU168236

TU158486 
Polytrichum commune Hedw.

fq $\quad$ LC

var. commune

TU168470

var. periogonale (Michx.) Hampe

TU167304

Polytrichum formosum Hedw.

Polytrichum juniperinum Hedw.

Polytrichum longisetum Sw. ex Brid.

Polytrichum pallidisetum Funck

Polytrichum piliferum Hedw.

Polytrichum strictum Menzies ex Brid.

Pseudephemerum nitidum (Hedw.) Loeske

Pseudoamblystegium subtile (Hedw.) Vanderp. \& Hedenäs

Pseudobryum cinclidioides (Huebener) T.J.Kop.

Pseudocampylium radicale (P.Beauv.) Vanderp. \& Hedenäs

Pseudocrossidium hornschuchianum (Schultz) R.H.Zander

Pseudocrossidium revolutum (Brid.) R.H.Zander

Pseudoleskeella catenulata (Brid. ex Schrad.) Kindb.

Pseudoleskeella nervosa (Brid.) Nyholm

Pseudoscleropodium purum (Hedw.) M.Fleisch.

Pseudotaxiphyllum elegans (Brid.) Z.Iwats.

Pterigynandrum filiforme Hedw.

var. filiforme

$\begin{array}{lll} & & \text { TU168470 } \\ & & \text { TU167304 } \\ \text { fq } & & \text { TU169777 } \\ \text { fq } & \text { LC } & \text { TU161401 } \\ \text { fq } & & \text { TU167342 } \\ \text { r } & \text { VU } & \text { TU167255 } \\ \text { fq } & \text { LC } & \text { TU167728 } \\ \text { fq } & \text { LC } & \text { TU169341 } \\ \text { ? } & \text { RE } & \text { TAA5004585 } \\ \text { fq } & & \text { TU152501 } \\ \text { p } & & \text { TU169184 } \\ \text { r } & \text { VU } & \text { TU161303 } \\ \text { p } & \text { NT } & \text { TAM B737:179 } \\ \text { r } & & \text { TU150822 } \\ \text { p } & \text { NT } & \text { TU158921 } \\ \text { fq } & \text { LC } & \text { TU152807 } \\ \text { fq } & & \text { TAM B851:16 } \\ \text { r } & & \text { TU167298 }\end{array}$

var. majus (De Not.) De Not.

TU161296

TU158925

Ptilium crista-castrensis (Hedw.) De Not.

Ptychostomum archangelicum (Bruch \& Schimp.) J.R.Spence

Ptychostomum arcticum (R.Br.) J.R.Spence ex D.T.Holyoak \& N.Pedersen

$\mathrm{p}$

Ptychostomum boreale (F.Weber \& D Mohr) Ochyra \& Bednarek-Ochyra

Ptychostomum capillare (Hedw.) D.T.Holyoak \& N.Pedersen

Ptychostomum cernuum (Hedw.) Hornsch.

Ptychostomum compactum Hornsch.

Ptychostomum imbricatulum (Müll.Hal) D.T.Holyoak \& N.Pedersen

Ptychostomum moravicum (Podp.) Ros. \& Mazimpaka

Ptychostomum pallens (Sw.) J.R.Spence

Ptychostomum pseudotriquetrum (Hedw.) J.R.Spence \& H.P.Ramsay

var. pseudotriquetrum

var. bimum (Schreb.) D.T.Holyoak \& N.Pedersen

Ptychostomum rubens (Mitt.) D.T.Holyoak \& N.Pedersen

Pylaisia polyantha (Hedw.) Schimp.

Racomitrium aciculare (Hedw.) Brid.

Racomitrium canescens (Hedw.) Brid.

Racomitrium elongatum Ehrh. ex Frisvoll

Racomitrium ericoides (Brid.) Brid.

Racomitrium fasciculare (Hedw.) Brid.

fq $\quad$ LC

TU169340

TU158508

TU158503

TAM B773:56

TU169287

TAM B743:34

TAM B823:141

$\mathrm{fq}$

fq LC

TU161302

$\mathrm{fq}$

TU152886

TU160977

fq LC

TU169257

TAA5001249

TU169457

TU151027

TAA5004593

TAM0070063

TAM B868:112

TAM B868:177

TAM B774:111

TAM B772:191

Racomitrium heterostichum (Hedw.) Brid.

TAM0074053

Racomitrium lanuginosum (Hedw.) Brid.

TAM B772:194
TAM B817:75

Racomitrium microcarpon (Hedw.) Brid.

Racomitrium sudeticum (Funck) Bruch \& Schimp. 
TU167369

Rhizomnium pseudopunctatum (Bruch \& Schimp.) T.J.Kop.

Rhizomnium punctatum (Hedw.) T.J.Kop.

$\mathrm{p}$

fq $\quad$ LC

Rhodobryum ontariense (Kindb.) Kindb.

Rhodobryum roseum (Hedw.) Limpr.

Rhynchostegium murale (Hedw.) Schimp.

Rhynchostegium riparioides (Hedw.) Cardot

Rhytidiadelphus loreus (Hedw.) Warnst.

Rhytidiadelphus squarrosus (Hedw.) Warnst.

Rhytidiadelphus subpinnatus (Lindb.) T.J.Kop.

Rhytidiadelphus triquetrus (Hedw.) Warnst.

Rhytidium rugosum (Hedw.) Kindb.

Saelania glaucescens (Hedw.) Broth.

Sanionia uncinata (Hedw.) Loeske

Sarmentypnum exannulatum (Schimp.) Hedenäs

Schistidium agassizii Sull. \& Lesq.

Schistidium apocarpum (Hedw.) Bruch \& Schimp.

Schistidium confertum (Funck) Bruch \& Schimp.

Schistidium confusum H.H.Blom

Schistidium crassipilum H.H.Blom

Schistidium elegantulum H.H.Blom

Schistidium maritimum (Sm. ex R.Scott) Bruch \& Schimp.

subsp. maritimum

subsp. piliferum (I.Hagen) B.Bremer

Schistidium papillosum Culm.

Schistidium platyphyllum (Mitt.) H.Perss.

Schistidium rivulare (Brid.) Podp.

Schistidium robustum (Nees \& Hornsch.) H.H.Blom

Schistidium submuticum H.H.Blom

Schistidium trichodon (Brid.) Poelt

Schistostega pennata (Hedw.) F.Weber \& D.Mohr.

Sciuro-hypnum curtum (Lindb.) Ignatov

Sciuro-hypnum plumosum (Hedw.) Ignatov \& Huttunen, nom. cons.

Sciuro-hypnum populeum (Hedw.) Ignatov \& Huttunen

Sciuro-hypnum reflexum (Starke) Ignatov \& Huttunen

Sciuro-hypnum starkei (Brid.) Ignatov \& Huttunen

Scorpidium cossonii (Schimp.) Hedenäs

Scorpidium revolvens (Sw. ex anon.) Rubers

Scorpidium scorpioides (Hedw.) Limpr.

Seligeria calcarea (Hedw.) Bruch \& Schimp.

Seligeria campylopoda Kindb.

Seligeria donniana (Sm.) Müll.Hal.

Seligeria patula (Lindb.) I.Hagen

Seligeria pusilla (Hedw.) Bruch \& Schimp.

Seligeria recurvata (Hedw.) Bruch \& Schimp.

Serpoleskea confervoides (Brid.) Loeske

Sphagnum angustifolium (C.E.O.Jensen ex Russow) C.E.O.Jensen

Sphagnum aongstroemii C.Hartm.

$$
\mathrm{p}
$$$$
f_{q}
$$$$
\mathrm{r}
$$$$
\text { fq }
$$$$
r
$$

$\mathrm{p}$

r

$r$

p

VU

p

$\mathrm{p}$
TU168577

TU169939

TU168688

TAM B876:1270

TU159837

TU167119

TU169070

TU169281

TU168458

TU169250

TAA5000883

TU152513

TU168702

TAA5004591

TAM B837:192

TAM 808:136

TAM B828:47

TAM B796:295

TAM B753:52

TAM B836:77

TAM B813:36

TAM B838:10

TAM B740:171

TAM B836:76

TAM B753:54

TAM B868:279

TAM B858:167

TAA5003525

TAM0070232

TALL D003271

TAM B876:32

TAM B866:270

TAM B872:319

TU161262

TU161268

TU167743

TU167197

TU168228

TAA5004616

TAM0076942

TU151875

TAA5003579

TU162002

TU168382

TU168767 
Sphagnum auriculatum Schimp.

DD

TU169438

TU151000

Sphagnum austinii Sull.

Sphagnum balticum (Russow) C.E.O.Jensen

Sphagnum capillifolium (Ehrh.) Hedw.

Sphagnum centrale C.E.O.Jensen

Sphagnum compactum Lam. \& DC.

Sphagnum contortum Schultz

Sphagnum cuspidatum Ehrh. ex Hoffm.

Sphagnum fallax (H.Klinggr.) H.Klinggr.

Sphagnum fimbriatum Wilson

Sphagnum flexuosum Dozy \& Molk.

Sphagnum fuscum (Schimp.) H.Klinggr.

Sphagnum girgensohnii Russow

Sphagnum inundatum Russow

Sphagnum jensenii H.Lindb.

Sphagnum lindbergii Schimp.

Sphagnum magellanicum Brid.

Sphagnum majus (Russow) C.E.O.Jensen

subsp. majus

r

$\mathrm{fq}$

$\mathrm{fq}$

$\mathrm{fq}$

$\mathrm{fq}$

$\mathrm{fq}$

fq

fq

fq

fq

fo

fq

fq

$\mathrm{p}$

$\mathrm{r}$

$\mathrm{p}$

$\mathrm{fq}$

fq

subsp. norvegicum Flatberg

Sphagnum molle Sull.

Sphagnum obtusum Warnst.

Sphagnum palustre L.

Sphagnum papillosum Lindb.

Sphagnum platyphyllum (Lindb. ex Braithw.) Warnst.

Sphagnum pulchrum (Lindb. ex Braithw.) Warnst.

Sphagnum quinquefarium (Braithw.) Warnst.

Sphagnum riparium Ångstr.

Sphagnum rubellum Wilson

Sphagnum russowii Warnst.

Sphagnum squarrosum Crome

Sphagnum subfulvum Sjors

Sphagnum subnitens Russow \& Warnst.

Sphagnum subsecundum Nees

Sphagnum tenellum (Brid.) Pers. ex Brid.

Sphagnum teres (Schimp.) Ångstr.

Sphagnum warnstorfii Russow

Sphagnum wulfianum Girg.

Splachnum ampullaceum Hedw.

Splachnum rubrum Hedw.

Splachnum sphaericum Hedw.

Splachnum vasculosum Hedw.

Straminergon stramineum (Dicks. ex. Brid.) Hedenäs

Syntrichia calcicola J.J.Amann

Syntrichia caninervis Mitt.

var. astrakhanica Ignatov, Ignatova \& Suragina

Syntrichia montana Nees
TU168666

TU168527

TU169390

TU168389

TU168498

TU169171

TU160935

TU168493

TU168399

TU168487

TU168524

TU168074

TAA5004617

TU168402

TU168386

TU151773

TU168616

TU150810

TU168593

TU169392

TU168621

TU168397

TU168754

TU150799

TU169422

TU168486

TU169429

TU168387

TU168651

TU169272

TU168659

TU160918

TU152630

TU168526

TU168638

TU169410

TU150910

TU151532

TU151533

TU168653

TAA5000888

TAA5000890

TAA5000891 
Syntrichia norvegica F.Weber

Syntrichia ruralis (Hedw.) F.Weber \& D.Mohr

var. ruralis

var. ruraliformis (Besch.) Delogne

Syntrichia virescens (De Not.) Ochyra

Taxiphyllum wissgrillii (Garov.) Wijk \& Margad.

Tayloria tenuis (Dicks.) Schimp.

Tetraphis pellucida Hedw.

Tetraplodon mniodes (Hedw.) Bruch \& Schimp.

Thamnobryum alopecurum (Hedw.) Gangulee

Thamnobryum neckeroides (Hook.) E.Lawton

Thamnobryum subserratum (Hook. ex Harv.) Nog. \& Z.Iwats.

Thuidium assimile (Mitt.) A.Jaeger

Thuidium delicatulum (Hedw.) Schimp.

Thuidium recognitum (Hedw.) Lindb.

Thuidium tamariscinum (Hedw.) Schimp.

Timmia bavarica Hessl.

Timmia megapolitana Hedw.

Tomentypnum nitens (Hedw.) Loeske

Tortella fragilis (Hook. \& Wilson) Limpr.

Tortella inclinata (R.Hedw.) Limpr.

var. inclinata

var. densa (Lorentz \& Molendo) Limpr.

Tortella rigens Alberts.

Tortella tortuosa (Hedw.) Limpr.

var. tortuosa

var. fragilifolia (Jur.) Limpr.

Tortula acaulon (With.) R.H.Zander

var. acaulon

var. piliferum (Hedw.) Hook. \& Taylor

Tortula caucasica Lindb.

Tortula lindbergii Broth.

Tortula lingulata Lindb.

Tortula muralis Hedw.

Tortula protobryoides R.H.Zander

Tortula randii (Kenn.) R.H.Zander

Tortula schimperi M.J.Cano, O.Werner \& J.Guerra

Tortula subulata Hedw.

Tortula truncata (Hedw.) Mitt.

Trematodon ambiguus (Hedw.) Hornsch.

Trichodon cylindricus (Hedw.) Schimp.

Trichostomum brachydontium Bruch

Trichostomum crispulum Bruch

Ulota bruchii Hornsch. ex Brid.

Ulota coarctata (P.Beauv.) Hammar

Ulota crispa (Hedw.) Brid.

Ulota curvifolia (Wahlenb.) Lilj. fq

$\mathrm{p}$

r

fq

$r$

$\mathrm{p}$

r

fo

$\mathrm{f}$

fq

fq

$\mathrm{p}$

$r$

$r$

$\mathrm{fq}$

$\mathrm{fq}$

$\mathrm{fq}$

p VU

fq LC

VU II

EN

LC

DD

NT III

III

TAM B767:205

TU167925

TAM B753:43

TU167926

TAA5003733

TAA5003753

TAA5003771

TAA5003778

TU158497

TU168003

TU169273

TU168247

TU169359

TU169356

TU169360

TU169437

TU169354

$\mathrm{p}$

TAA5000870

TAA5000866

TAA5000933

S; reg.no.B216405

TAA5004587

TAA5003834

TAM B821:182

TU170670

TAM B803:37

TAA5003836

TAA5000943

TAA5000953

TAA5000686

TAA5004596

TAM0070261

TAA5003869

TAA5004588

TAA5003879

TAA5000955 
Ulota drummondii (Hook. \& Grev.) Brid.

Ulota hutchinsiae (Sm.) Hammar

Warnstorfia fluitans (Hedw.) Loeske

Warnstorfia trichophylla (Warnst.) Tuom. \& T.J.Kop.

Warnstorfia tundrae (Arnell) Loeske

Weissia brachycarpa (Nees \& Hornsch.) Jur.

Weissia controversa Hedw.

Weissia squarrosa (Nees \& Hornsch.) Müll.Hal.

Zygodon rupestris Schimp. ex Lorentz

Zygodon stirtonii Schimp. ex Stirt.

Zygodon viridissimus (Dicks.) Brid.

$\begin{array}{lll}\mathrm{r} & \mathrm{VU} & \text { TAA5000961 } \\ \mathrm{r} & \mathrm{VU} & \text { TAA5000963 } \\ \mathrm{fq} & & \text { TU151008 } \\ \mathrm{p} & \mathrm{LC} & \mathrm{TU} 169461 \\ \mathrm{r} & \mathrm{VU} & \mathrm{TU} 168412 \\ \mathrm{p} & & \text { TAA5000967 } \\ \mathrm{p} & & \text { TAA5004589 } \\ \mathrm{r} & \mathrm{NT} & \text { TAA5000990 } \\ \mathrm{r} & & \text { TAA5000907 } \\ \mathrm{r} & & \text { TAA5000908 } \\ \mathrm{p} & \mathrm{NT} & \text { TAA5000911 }\end{array}$

Hodgetts, N.G. 2015. Checklist and country status of European bryophytes - toward a new Red List of Europe. Irish Wildlife Manuals 84: 1-125.

We are thankful to Lars Hedenäs and Jiři Váňa for helping with voucher specimens localization. Leiti Kannukene and Merlyn Pajur helped us with specimens from TALL. This work has been supported by the following research projects: IUT20-29, IUT34-7, state program KOGUD for botanical collections of EULS and NHM TU, and by the EU Regional Development Fund (Centre of Excellence FIBIR).

\section{REFERENCES}

Blom. H.H. 1996. A revision of the Schistidium apocarpum complex in Norway and Sweden. Bryophytorum Bibliotheca 49: 1-320.

Feldberg, K., Váňa, J., Hentschel, J. \& Heinrichs, J. 2010. Currently accepted species and new combinations in Jamesonielloideae (Adelanthaceae, Jungermanniales). Cryptogamie, Bryologie 31(2): 141-146.

Frey, W. \& Stech, M. 2009. Marchantiophyta, Bryophyta, Anthocerotophyta. In: Frey, W. (ed) Syllabus of Plant families. Adolf Engler's Syllabus der Pflanzenfamilien. Part 3. Bryophytes and seedless vascular plants. Gebrüder Borntraeger, Berlin Stuttgart. Pp. 9-264.

Girgensohn, G.K. 1860. Naturgeschichte der Laubund Lebermoose Liv-, Ehst- und Kurlands. Arch. Naturk. Liv-, Ehst- und Kurlands 2(2): 1-488.

Hallinbäck, T. 2002. Globally widespread bryophytes, but rare in Europe. Portugaliae Acta Biol. 20: $11-24$.

Hassel, K. \& Söderström, L. 2005. The expansion of the alien mosses Orthodontium lineare and Campylopus introflexus in Britain and continental Europe. J. Hattori Bot. Lab. 97: 183-193.

Hedenäs, L. 1990. Additions to the moss floras of Estonia and Latvia. Lindbergia 16: 113-114.

Hedenäs, L. \& Rosborg, C. 2008. Pseudocalliergon is nested within Drepanocaldus (Bryophyta: Amblystegiaceae). Lindbergia 33: 67-74.
Holyoak, D.T. \& Hedenäs, L. 2006. Morphological, ecological and molecular studies of the integrading taxa Bryum neodamense and $B$. pseudotriquetrum (Bryopsida: Bryaceae). Journal of Bryology 28: 299-311. http://dx.doi. org/10.1179/174328206X136304

Ingerpuu, N., Kalda, A., Kannukene, L., Krall, H., Leis, M. \& Vellak, K. 1994. Eesti sammalde nimestik. List of the Estonian bryophytes. Abiks Loodusevaatlejale 94: 1-175.

Kannukene, L., Ingerpuu, N., Vellak, K. \& Leis, M. 1997. Additions and amendments to the list of Estonian bryophytes. Folia Cryptogamica Estonica 31: 1-7.

Leis, M. \& Kannukene, L. 2007. New Estonian Records: mosses. Folia Cryptogamica Estonica 43: 69-72.

Malta, N. 1930. Übersicht der Moosflora des Ostbaltischen Gebietes II. Laubmoose (Andreales et Bryales). Acta Horti Botanici Universitatis Latviensis 1/3: 75-184.

Riigi Teataja 1994. Kaitstavate loodusobjektide seadus. Vastu võetud 01.01.1994. RT I 1994, 46,773. (in Estonian)

Riigi Teataja 2014a. I ja II kaitsekategooriana kaitse alla võetavate liikide loetelu. VV. RT I, 18.06.2014, 20. (in Estonian)

Riigi Teataja 2014b. III kaitsekategooria liikide kaitse alla võtmine. KKM, RT I, 04.07.2014, 22. (in Estonian)

Shaw, J., Szövényi, P. \& Shaw, B. 2011. Bryophyte diversity and evolution: Windows into the early evolution of land plants. American Journal of Botany 98(3): 352-369. http://dx.doi.org/10.3732/ ajb. 1000316

Shaw, B., Crandall-Stotler, B., Váňa, J., Stotler, R. E., von Konrat, M., Engel, J.J., Davis, E.K., Long, D.G. Sova, P. \& Shaw, A.J. 2015. Phylogenetic relationships and morphological evolution in a major clade of leafy liverworts (Phylum Marchantiophyta, Order Jungermanniales): Suborder Jun- 
germanniineae. Systematic Botany 40(1): 27-45. http://dx.doi.org/10.1600/036364415X686314 Spence, J.R. 2005. New genera and combinations in Bryaceae (Bryales, Musci) for North America. Phytotaxa 87(1): 15-28.

Söderström, L., De Roo, R. \& Hedderson, T. 2010. Taxonomic novelties resulting from recent reclassification of the Lophoziaceae/Scapaniaceae clade. Phytotaxa 3: 47-53.

Söderström, L., Váňa, J., Crandall-Stotler, B., Renner, M.A.M., Hagborgs A. \& von Konrat, M. 2015. Notes on Early Land Plants Today. 68. Miscellaneous notes on Marchantiophyta. Phytotaxa 202(1): 69-72. http://dx.doi.org/10.11646/ phytotaxa.202.1.10

Váňa, J. 1973. Studien über die Jungermannioideae (Hepaticae). 3. Jungermannia Subg. Liochlaena. Folia Geobot. Phytotax. 8: 397-416. http://dx.doi. org/ 10.1007/BF02852064

Váňa, J., Söderström, L., Hagborg, A. \& von Konrat, M.J. 2013. Notes on Early Land Plants Today. 40. Notes on Cephaloziellaceae (Marchantiophyta). Phytotaxa 112: 1-6. http://dx.doi.org/10.11646/ phytotaxa.112.1.1

Vanderpoorten, A. \& Goffinet, B. 2009. Introduction to bryophytes. Cambridge University Press, Cambridge, UK. 296 pp. http://dx.doi.org/10.1017/ CBO9780511626838

Vellak, K. \& Ingerpuu, N. 2012. The status of bryophyte conservation in Estonia. Studia botanica hungarica 43: 59-68.
Vellak, K., Ingerpuu, N., Kannukene, L. \& Leis, M. 2006. New Estonian records. Liverworts and mosses. Folia Cryptogamica Estonica 42: 107-111.

Vellak, K., Ingerpuu, N., Kannukene, L. \& Leis, M. 2009. New Estonian records and amendments. Liverworts and mosses. Folia Cryptogamica Estonica 45: 91-93.

Vellak, K., Kannukene, L., Ingerpuu, N. \& Leis, M. 2001. Additions to the list of the Estonian bryophytes, 1997-2001. Folia Cryptogamica Estonica 38: 71-78.

Vellak, K., Kannukene, L., Leis, M. \& Ingerpuu, N. 2013. New Estonia records: Mosses. Folia Cryptogamica Estonica 50: 121-122. http://dx.doi. org/ 10.12697/fce.2013.50.15

Vellak, K., Leis, M., Ingerpuu, N. \& Kannukene, L. 2011. New Estonia records: Mosses. Folia Cryptogamica Estonica 48: 153-158.

Von Konrat, M., Söderström, L., Renner, M.A.M., Hagborg, A., Briscoe, L. \& Engel, J.J. 2010. Early land plants today (ELPT): How many liverworts species are there? Phytotaxa 9: 22-40. http:// dx.doi.org/10.11646/phytotaxa.9.1.5

Werner, O., Ros, R.M., Cano, M.J. \& Guerra, J., 2004 Molecular phylogeny of Pottiaceae (Musci) based on chloroplast rps4 sequence data. Plant Systematics and Evolution 243: 147-164. http://dx.doi. org/10.1007/s00606-003-0076-0 
128 Folia Cryptog. Estonica 